\title{
Higher order multipole moments for molecular dynamics simulations
}

\author{
Nuria Plattner • Markus Meuwly
}

Received: 16 September 2008 / Accepted: 6 January 2009/Published online: 5 March 2009

(C) Springer-Verlag 2009

\begin{abstract}
In conventional force fields, the electrostatic potential is represented by atom-centred point charges. This choice is in principle arbitrary, but technically convenient. Point charges can be understood as the first term of multipole expansions, which converge with an increasing number of terms towards the accurate representation of the molecular potential given by the electron density distribution. The use of multipole expansions can therefore improve the force field accuracy. Technically, the implementation of atomic multipoles is more involved than the use of point charges. Important points to consider are the orientation of the multipole moments during the trajectory, conformational dependence of the atomic moments and stability of the simulations which are discussed here.
\end{abstract}

Keywords Multipole moments · Molecular dynamics ·

Point charges · Electron density distribution .

Distributed multipole analysis .

Cumulative atomic multipole moments .

Theory of atoms in molecules

\section{Introduction}

In atomistic force fields such as CHARMM or AMBER $[12,26]$, the electrostatic potential is usually represented by point charges. Charges centred on an atom are not an intrinsic physical property but rather a convenient concept, and are typically obtained from a population analysis of the Hartree-Fock wave function from electronic structure

N. Plattner $\cdot$ M. Meuwly $(\bowtie)$

Department of Chemistry, University of Basel,

4056 Basel, Switzerland

e-mail: m.meuwly@unibas.ch calculations [13], or by fitting atomic charges that optimally reproduce the electrostatic potential or interaction energies $[2,20]$. The physical property from which charges are derived is the molecular electron density $\rho(r)$, where $r$ is a point in three-dimensional (3D) space. It has been demonstrated in the past that point charges can be understood as a first term in a multipole expansion [21, 22]. The potential that corresponds to the multipole expansion converges towards the potential derived from $\rho(r)$ on which the analysis is based. Several algorithms have been developed for obtaining atom centred multipole expansions. The most widely used are distributed multipole analysis (DMA) [25], cumulative atomic multipole moments (CAMM) [21] and the theory of atoms in molecules (AIM) [1]. All these methods include a partitioning of the electron density and an integration of $\rho(r)$ over a suitably defined space occupied by a particular atom for all desired multipole ranks. The partitioning of $\rho(r)$, the allocation of atomic moments to particular atoms, and therefore the resulting multipole expansion, its convergence, basis set dependence, conformational dependence and transferability differ between the three methods. A direct comparison of DMA (in its previous form [22]) and AIM has been published [7].

Atomic multipole moments have been found to be important for modelling molecular interactions in complexes, solids and proteins [17]. In particular, they have been applied successfully to organic crystal structure prediction [3], and they have also been shown to improve results of molecular dynamics (MD) simulations for several systems [14-16].

In the following, practical issues in using atomic multipole moments in MD simulations are considered. The properties of electrostatic multipoles are compared to different point charge models and specific problems concerning the implementation of atomic multipoles in the 

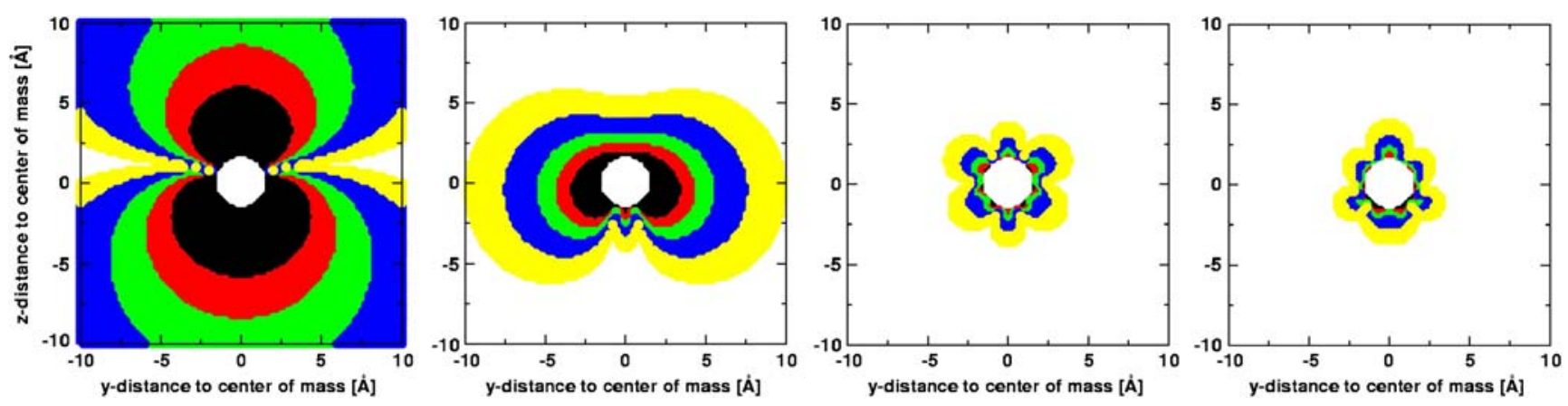

Fig. 1 Differences (absolute values) between $\phi_{\rho}$ (potential from multipole expansion) and $\phi$ (potential from ab initio calculations) for water, based on a Hartree-Fock calculation with a $6-31 G^{*}$ basis set.

context of MD simulations are then analysed and the effects demonstrated for model systems.

\section{Choice of atomic multipole parameters for MD simulations}

The electrostatic energy of a multipole expansion up to quadrupole on two atoms $a$ and $b$ in spherical tensor notation can be written as

$$
\begin{aligned}
E_{\text {elec }}= & E\left(q_{a} q_{b}\right)+\sum_{k=1}^{3} E\left(q_{a} \mu_{b}^{k}\right)+\sum_{l=1}^{3} E\left(\mu_{a}^{l} q_{b}\right) \\
& +\sum_{k=1}^{3} \sum_{l=1}^{3} E\left(\mu_{a}^{l} \mu_{b}^{k}\right)+\sum_{m=1}^{5} E\left(q_{a} \Theta_{b}^{m}\right)+\sum_{n=1}^{5} E\left(\Theta_{a}^{n} q_{b}\right) \\
& +\sum_{k=1}^{3} \sum_{m=1}^{5} E\left(\mu_{a}^{k} \Theta_{b}^{m}\right)+\sum_{n=1}^{5} \sum_{l=1}^{3} E\left(\Theta_{a}^{n} \mu_{b}^{k}\right) \\
& +\sum_{m=1}^{5} \sum_{n=1}^{5} E\left(\Theta_{a}^{n} \Theta_{b}^{m}\right)
\end{aligned}
$$

where $q_{a}$ is the charge of atom $a, \mu_{b}^{k}$ is the direction $k$ of the dipole moment vector on atom $b, \Theta_{a}^{n}$ is the element $n$ of the quadrupole tensor on atom $a$ [23]. As an example, the interaction between a charge $q_{a}$ and the $\Theta_{b}^{20}$ quadrupole tensor element is given by

$E\left(q_{a} \theta_{b}^{20}\right)=q_{a} \Theta_{b}^{20} \frac{1}{2} \frac{\left(3\left(r_{z}^{b}\right)^{2}-1\right)}{R^{3}}$,

where $r_{z}^{b}$ and is the $z$-element of a unit vector pointing from $b$ to $a$ and $R$ is the interatomic distance.

The practical use of atomic multipole moments for MD simulations should take a number of considerations into account. First, to justify the additional computational cost, the electrostatic potential derived from the multipole expansion has to be significantly more accurate than a corresponding point charge potential. Second, the compu-
From left to right: distributed multipoles up to rank 0, rank 1, rank 2 and rank 3. Colour coding: black $>10 \mathrm{~kJ} / \mathrm{mol}$, red $5-10 \mathrm{~kJ} / \mathrm{mol}$, green $2.5-$ $5 \mathrm{~kJ} / \mathrm{mol}$, blue $1-2.5 \mathrm{~kJ} / \mathrm{mol}$, yellow $0.5-1 \mathrm{~kJ} / \mathrm{mol}$, white $<0.5 \mathrm{~kJ} / \mathrm{mol}$

tational expense depends sensitively on the multipole rank at which the expansion can be truncated with only small remaining differences between the exact electrostatic potential and the potential from the multipole expansion. Third, the accuracy of the electrostatics inside the van der Waals radii is of less importance since at very short range the interaction is dominated by the repulsive part of the Lennard-Jones (LJ) potential. Fourth, for the parametrisation of larger molecules, the atomic multipole moments should be transferable between smaller fragments without large errors (e.g. transferability between amino acids in different peptides). Finally, the change of the multipole moments with molecular conformation should either be small or should be described by an analytical function. In the following, the properties of atomic moments obtained from DMA [24] are analysed, using alanine and glycine as examples, covering the points mentioned above.

\section{Convergence and accuracy of distributed multipole} potentials

For all the molecules and ab initio methods studied, the distributed multipole expansion converges to within $10 \%$ at rank two (i.e. quadrupole). To illustrate this, Fig. 1 shows the difference between potential $\phi_{\rho}$ (derived from the multipole expansion) and the ab initio potential $\phi$ for water for different levels of accuracy. At rank two, $\phi_{\rho}$ typically differs by a few percent on average from $\phi$, and is significantly more accurate than potentials from point charges for the same molecule, using Mulliken charges or charges fitted according to the Merz-Kollman scheme [2]. Quantitative evaluations for water and alanine are shown in Table 1 . The evaluation is carried out with a probe charge of 1 , based on a cubic grid of $10 \times 10 \times 10 \AA$ for water and $15 \times 15 \times 15 \AA$ for alanine, with 101 points in each direction outside the van der Waals radii ${ }^{1}$.

\footnotetext{
${ }^{1}$ Multipole parameters identical to those from Table 2 for all molecules considered can be obtained from the authors on request.
} 
Table 1 Comparison for water and alanine. $H F$ Hartree-Fock, $D M A$ distributed multipole analysis

\begin{tabular}{|c|c|c|c|c|}
\hline & \multicolumn{2}{|c|}{ HF 6-31G* } & \multicolumn{2}{|c|}{ B3LYP Aug-cc-pVTZ } \\
\hline & Water & Alanine & Water & Alanine \\
\hline \multicolumn{5}{|l|}{ DMA, rank 0} \\
\hline$\Delta \bar{E}(\mathrm{~kJ} / \mathrm{mol})$ & 3.27 & 2.87 & 2.70 & 2.52 \\
\hline$\Delta \bar{E}(\%)$ & 64 & 2383 & 68 & 1653 \\
\hline \multicolumn{5}{|l|}{ DMA, rank 1} \\
\hline$\Delta \bar{E}(\mathrm{~kJ} / \mathrm{mol})$ & 0.63 & 0.333 & 0.71 & 0.36 \\
\hline$\Delta \bar{E}(\%)$ & 41 & 178 & 61 & 73 \\
\hline \multicolumn{5}{|l|}{ DMA, rank 2} \\
\hline$\Delta \bar{E}(\mathrm{~kJ} / \mathrm{mol})$ & 0.05 & 0.05 & 0.17 & 0.06 \\
\hline$\Delta \bar{E}(\%)$ & 1 & 6 & 4 & 8 \\
\hline \multicolumn{5}{|c|}{ Mulliken charges } \\
\hline$\Delta \bar{E}(\mathrm{~kJ} / \mathrm{mol})$ & 0.52 & 0.84 & 2.18 & 6.05 \\
\hline$\Delta \bar{E}(\%)$ & 23 & 687 & 60 & 2993 \\
\hline \multicolumn{5}{|l|}{ Merz-Kollman } \\
\hline$\Delta \bar{E}(\mathrm{~kJ} / \mathrm{mol})$ & 0.28 & 0.08 & 0.44 & 0.08 \\
\hline$\Delta \bar{E}(\%)$ & 18 & 35 & 37 & 18 \\
\hline
\end{tabular}

Conformational dependence of distributed multipole potentials

To illustrate the conformational dependence of multipolederived potentials, two conformations ( 1 and $\mathrm{c} 2$ ) of alanine and glycine, are considered (see Figs. 2, 3). Table 2 provides coordinates and multipole moments for glycine from which the potentials in Fig. 4 were calculated. This example shows that the differences between $\phi$ and $\phi_{\rho}$ are considerably larger if the multipoles are transferred to another conformation. A quantitative analysis of the differences between $\phi_{\rho}$ and $\phi$ is provided in Table 3, where differences between the electrostatic potentials from ab initio calculations and different charge models are reported. In order to calculate the data for another conformation, the multipoles are rotated according to the geometry of the atoms in the new conformation. The tables also show data for the conformational dependence for point charges, which are calculated according to the Merz-Kollmann scheme [2]. Also for point charges, the deviations in another conformation are considerably larger. The data show that the conformational dependence of $\phi_{\rho}$ has to be taken into account. Otherwise the accuracy gained by using multipole expansions instead of point charges is likely to be

Fig. 2 Alanine conformations $\mathrm{c} 1$ and $\mathrm{c} 2$<smiles>C[C](N)C(=O)O</smiles>

c1<smiles>C[C@H](N)C(=O)O</smiles>

c2

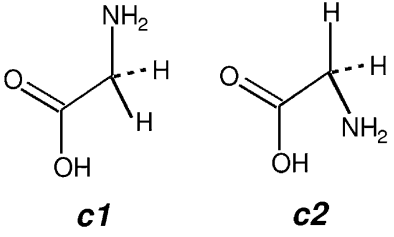

Fig. 3 Glycine conformations $\mathrm{c} 1$ and $\mathrm{c} 2$

compromised. The conformational dependence of $\phi_{\rho}$ has already been investigated in earlier studies for the DMA and for the CAMM algorithm [9, 18]. There are different possible solutions to this problem, not all of which are applicable in general.

For some molecules, the change of the atomic multipole moments with conformation can be described explicitly by an analytical function. This has been done successfully for CO [16]. Unlike this particularly simple case, more elaborate methods need to be used for molecules such as glycine and alanine. For the conformational dependence around torsion angles, the use of short Fourier series has been shown to significantly improve the electrostatic potential [10]. A combined solution for atomic multipoles together with inter- and intramolecular polarisation has been suggested [19], but no explicit dynamics has been carried out with this procedure for larger molecules such as amino acids. Furthermore, it is unclear how well this approach is able to reproduce atomic multipole moments of another conformation, since only the combined electrostatic plus polarisation energies have been evaluated and the individual contribution of each term to different conformations is not available.

Another possible solution is to use conformationally averaged multipole moments to better reproduce $\phi$ on average. This has been done here for glycine and alanine by shifting multipole moments with ranks higher than zero to fewer sites. Furthermore, the relative atomic radii for Lebedev integration in the distributed multipole analysis based on the self-consistent field (SCF) density from the corresponding calculation can be varied to minimise conformational dependence. The data are shown in Table 3. This is not a general solution, but the data presented here shows that, at least for the cases considered here, such a procedure reduces the error due to conformational changes.

Finally, it may also be possible to use an iterative scheme in which the multipole moments are recalculated periodically. Such an approach has already been proposed and tested for energy minimisations of crystal structures [8].

\section{Implementation in MD simulations}

For the implementation of atomic multipole moments into atomistic simulations, additional interaction terms and their 


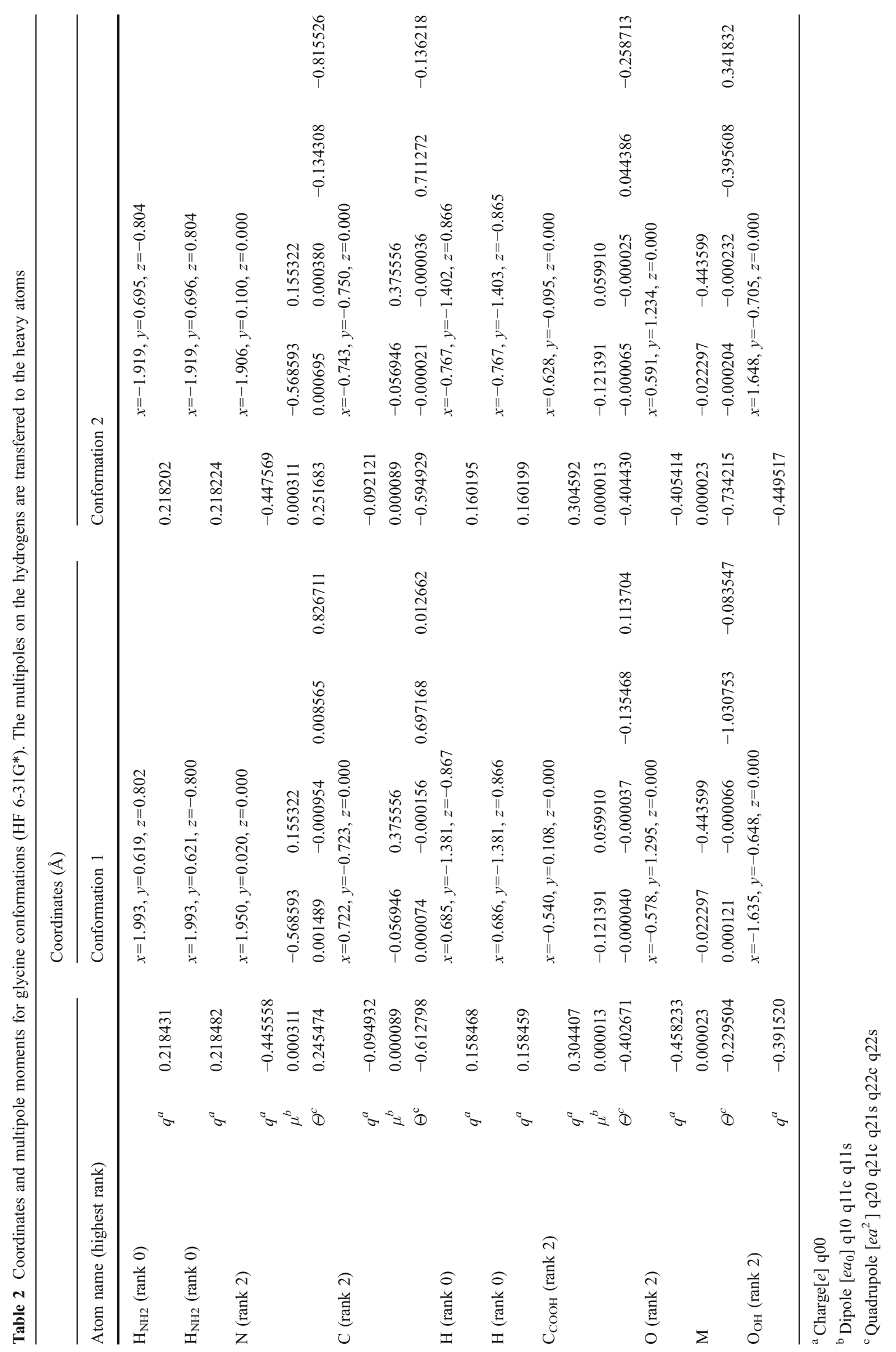


Fig. 4 Differences (absolute values) between multipole expansion and ab initio potential for glycine in two different conformations based on $\mathrm{HF}$ 6-31G* calculations. Upper row Multipole moments calculated from conformation 1 of glycine, lower row multipole moments calculated from conformation 2 of glycine. Left column Comparison with $\mathrm{ab}$ initio potential of conformation 1, right column comparison with $a b$ initio potential of conformation 2. The $\mathrm{x}$-axis of the second conformation is inverted in this representation in order to allow better comparison of the two conformations. Colour coding: Black $>10 \mathrm{~kJ} / \mathrm{mol}$, red $5-10 \mathrm{~kJ} / \mathrm{mol}$, green $2.5-5 \mathrm{~kJ} / \mathrm{mol}$, blue $1-2.5 \mathrm{~kJ} / \mathrm{mol}$, yellow $0.5-1 \mathrm{~kJ} / \mathrm{mol}$, white $<0.5 \mathrm{~kJ} / \mathrm{mol}$

Table 3 Conformational dependence of glycine and alanine; charges are calculated according to the Merz-Kollman scheme
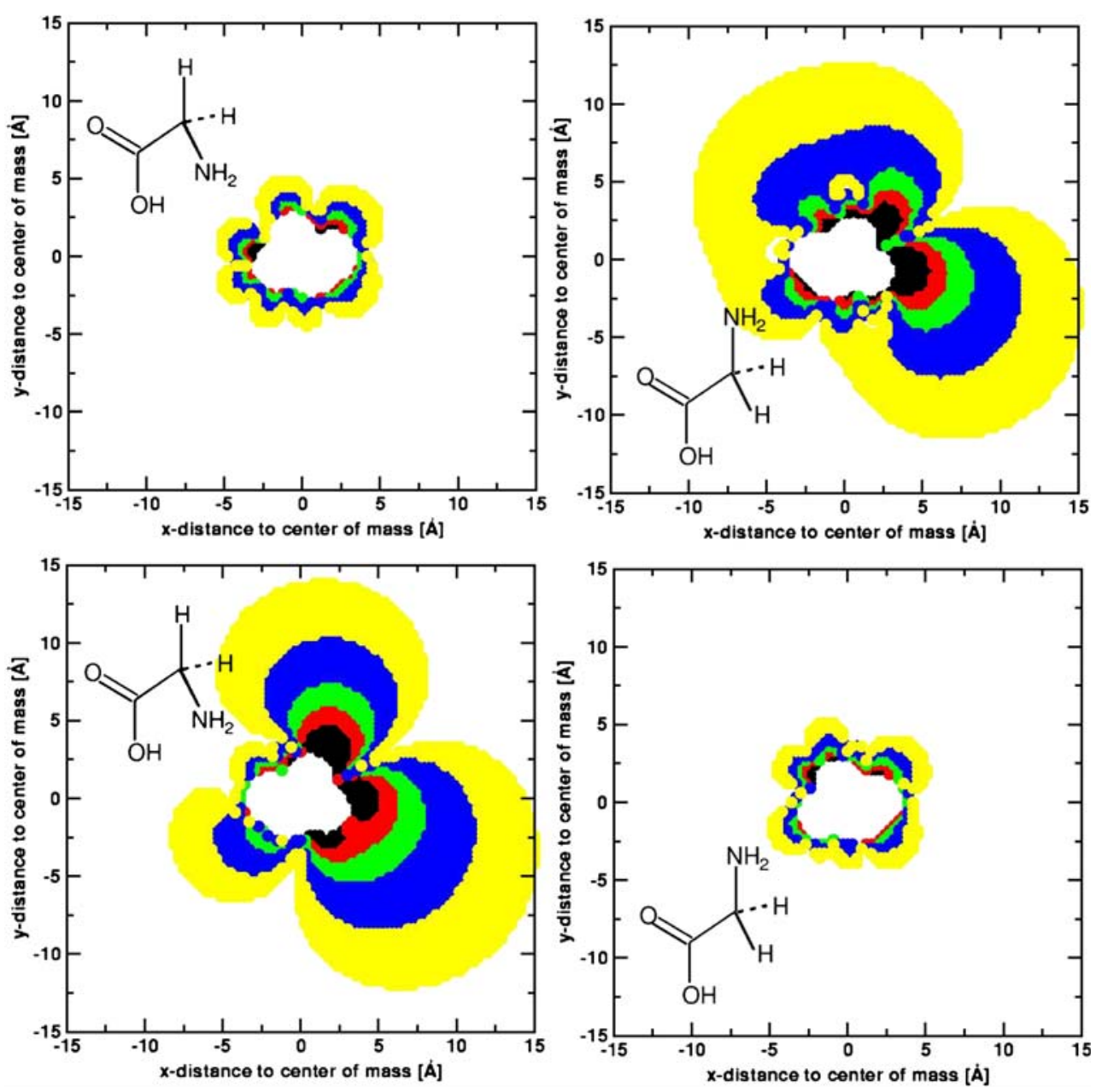

\begin{tabular}{|c|c|c|c|c|c|c|c|c|}
\hline & Glyci & & Alan & & Glyci & & Alan & \\
\hline & $\begin{array}{l}\mathrm{c} 1 \\
\mathrm{HF} 6\end{array}$ & $\begin{array}{c}\mathrm{c} 2 \\
\mathrm{~S}^{* *}\end{array}$ & $\begin{array}{l}\mathrm{cl} \\
\mathrm{HF} 6\end{array}$ & $\begin{array}{r}c 2 \\
\mathrm{G}^{* *}\end{array}$ & $\begin{array}{l}\mathrm{c} 1 \\
\text { B3LY }\end{array}$ & $\begin{array}{l}\mathrm{c} 2 \\
\mathrm{vQZZ}\end{array}$ & $\begin{array}{l}\text { c1 } \\
\text { B3L }\end{array}$ & $\begin{array}{c}\text { c2 } \\
\text { pVTZ }\end{array}$ \\
\hline Charges of $\mathrm{c} 1$ & & & & & & & & \\
\hline$\Delta \bar{E}(\mathrm{~kJ} / \mathrm{mol})$ & 0.09 & 0.53 & 0.07 & 1.03 & 0.11 & 0.44 & 0.08 & 1.05 \\
\hline$\Delta \bar{E}(\%)$ & 21 & 60 & 18 & 330 & 29 & 39 & 35 & 1317 \\
\hline Charges of $\mathrm{c} 2$ & & & & & & & & \\
\hline$\Delta \bar{E}(\mathrm{~kJ} / \mathrm{mol})$ & 0.17 & 0.15 & 0.75 & 0.08 & 0.17 & 0.15 & 0.47 & 0.10 \\
\hline$\Delta \bar{E}(\%)$ & 55 & 34 & 418 & 13 & 47 & 30 & 613 & 73 \\
\hline DMA of $\mathrm{c} 1$ & & & & & & & & \\
\hline$\Delta \bar{E}(\mathrm{~kJ} / \mathrm{mol})$ & 0.07 & 0.26 & 0.10 & 1.27 & 0.07 & 0.19 & 0.11 & 1.11 \\
\hline$\Delta \bar{E}(\%)$ & 4 & 30 & 11 & 295 & 5 & 25 & 15 & 829 \\
\hline DMA of $\mathrm{c} 2$ & & & & & & & & \\
\hline$\Delta \bar{E}(\mathrm{~kJ} / \mathrm{mol})$ & 0.16 & 0.07 & 0.63 & 0.10 & 0.14 & 0.11 & 0.54 & 0.11 \\
\hline$\Delta \bar{E}(\%)$ & 40 & 4 & 250 & 5 & 36 & 9 & 423 & 7 \\
\hline Setup with three & ultipole & es for & ine, fo & ites for & anine & & & \\
\hline DMA of $\mathrm{c} 1$ & & & & & & & & \\
\hline$\Delta \bar{E}(\mathrm{~kJ} / \mathrm{mol})$ & 0.10 & 0.12 & 0.10 & 0.32 & 0.10 & 0.13 & 0.10 & 0.46 \\
\hline$\Delta \bar{E}(\%)$ & 6 & 9 & 7 & 77 & 8 & 8 & 11 & 206 \\
\hline
\end{tabular}


first derivatives have to be provided. This can be done based on published interaction terms [23]. For the actual implementation it is important to take into account that higher order multipoles ( $k \geq 1$, i.e. dipole) depend on the coordinate system in which they were calculated. This can be handled through molecular reference axes systems and is considered in some more detail here. For distributed multipoles, moments obtained from GDMA [24] are defined with respect to the standard orientation of the molecule, obtained from the underlying GAUSSIAN [4] calculation. In a MD simulation the definitions of the multipole moments have to agree with the overall orientation of the corresponding molecule. Therefore, molecular reference axis systems have to be defined. In the simplest case (linear molecule) one reference axis, defined by the two atoms, is sufficient. For water, all three axes have to be defined. This can be done by aligning the $z$-axis along the axis pointing from the oxygen to the centre of the two hydrogens, the $y$-axis from one hydrogen to the other hydrogen, and the $x$-axis being then defined by orthogonality (see Fig. 5). For larger molecules, such as glycine and alanine, several reference axis systems have to be defined, since the different parts of the molecule can rotate with respect to each other. Finally, the multipole moments or the multipole interaction between molecules have to be rotated at each step of the simulation to the corresponding molecular reference axis system. This procedure ensures that the orientation of the atomic multipole moments, defined with respect to the equilibrium structure, agrees with the orientation of the molecule in space.

For molecular moments, the torques on the molecule can be calculated from the multipole interactions. This is not

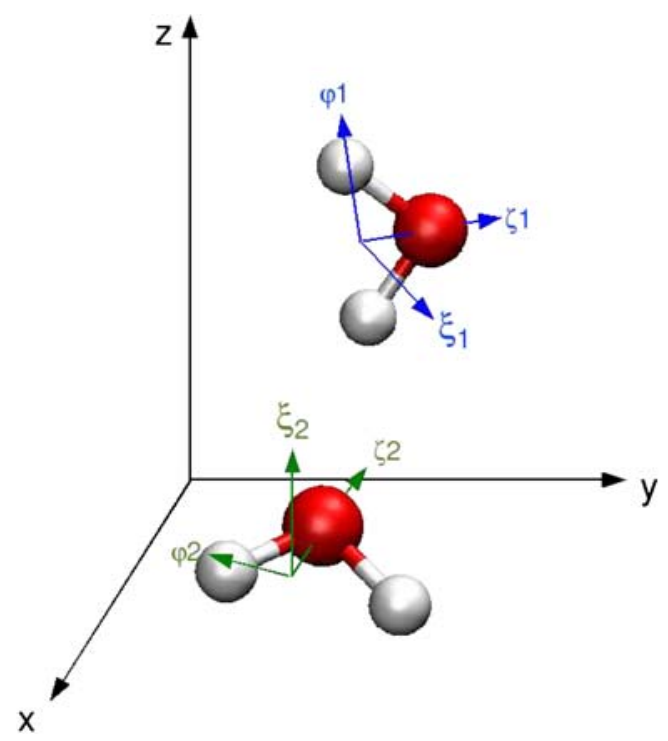

Fig. 5 Local reference axis systems for two water molecules in a global Cartesian coordinate system possible for atom-centred moments, as torques on point particles are not meaningful. For rigid molecules, the torque on the atoms can be transformed to torques on the molecules, which is not possible for flexible molecules. Therefore, molecular reference axis systems have to be used. Assigning a reference axis system is in principle a technical issue, since it corresponds to a limited number of symmetry groups from which all molecular geometries can be assembled. Nevertheless, an additional issue arises as the rotation of the atomic multipole moments slightly changes the interaction energy at each timestep because the reference axis system, in which the atomic multipoles are defined, rotates by a small amount between two consecutive time steps. The description of this rotation in terms of atom centred forces is difficult as, for each interacting pair of atoms, the forces depend not only on the relative position of these two atoms (as for all other force field terms), but also on the position of the other atoms belonging to the reference axis systems of that atom pair. Thus, in a rigorous analytical approach, additional forces will appear for all atoms that define the reference axis system. To the best of our knowledge no complete treatment is as yet available. If the overall orientational change at each step is small, these additional forces are small and can be neglected.

However, for cases where the energy contribution arising from the atomic multipoles is larger, the effect may become significant. The problem is different when the molecules are treated as rigid units, as done in the DL MULTI force field [5] where the forces are separated into a translational part (applied to the multipole sites) and a rotational part (applied to the molecular centre of mass) [11]. However, in this case the level of energy conservation depends on the choice of the electrostatic cutoffs. Furthermore, it has to be noted that this approach can be applied rigorously only to rigid molecules or rigid molecular fragments. It remains to be seen whether a generalisation of separating the rotational and translational part, or a more heuristic correction is preferable. In any case, for an approach that conserves energy, a combination of atomistic and molecular terms in the forces is required. One other way to circumvent this problem is to "absorb" the effect by using an NVT ensemble for which, however, careful testing of the simulation results is necessary to establish that no artifacts occur. It is particularly important that total energy is correctly partitioned between translational and rotational degrees of freedom according to the equipartition theorem.

\section{Practical applications in atomistic simulations}

As a test system to highlight the use of higher multipole moments in a realistic simulation, a mixed $\mathrm{CO} /$ water system is considered. The water molecules are described 


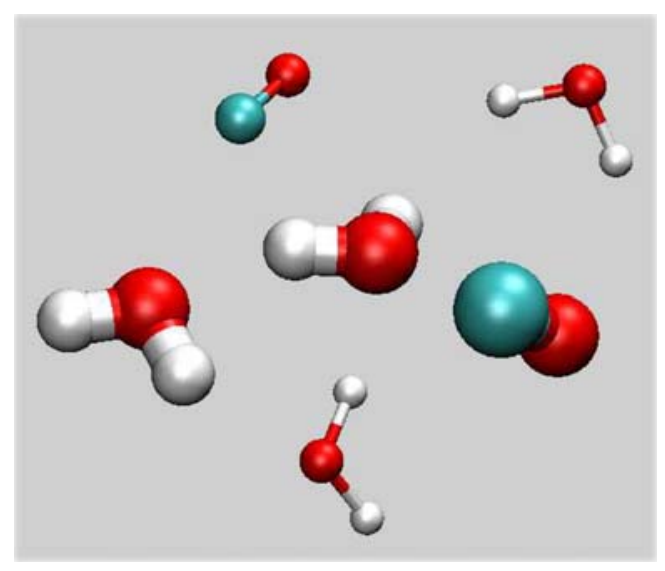

Fig. 6 Water/CO cluster structure. Molecules that are fixed during the trajectory are shown with smaller atom spheres, molecules that are free to move are shown with large atom spheres

by the TIP3P [6] potential, the $\mathrm{CO}$ molecules by different distributed multipole expansions that have already been used for $\mathrm{CO}$ in myoglobin and have been described in detail [16]. In the following, they will be referred to as Models B (multipoles up to rank 1 on carbon and up to rank 2 on oxygen), $\mathrm{C}$ (multipoles up to rank 2 on carbon and up to rank 3 on oxygen) and D (up to rank 3 on both atoms). Differences in the degree of energy conservation are found between rigid and flexible molecules, and between different parametrisations.

The first test system is a cluster of four water molecules and two $\mathrm{CO}$ molecules (see Fig. 6). The energy conservation is evaluated during $100 \mathrm{ps}$ at $5 \mathrm{~K}$, first for one $\mathrm{CO}$ in the static field of the other molecules, second for one $\mathrm{CO}$ and one water free to move, and third for one $\mathrm{CO}$ and two waters

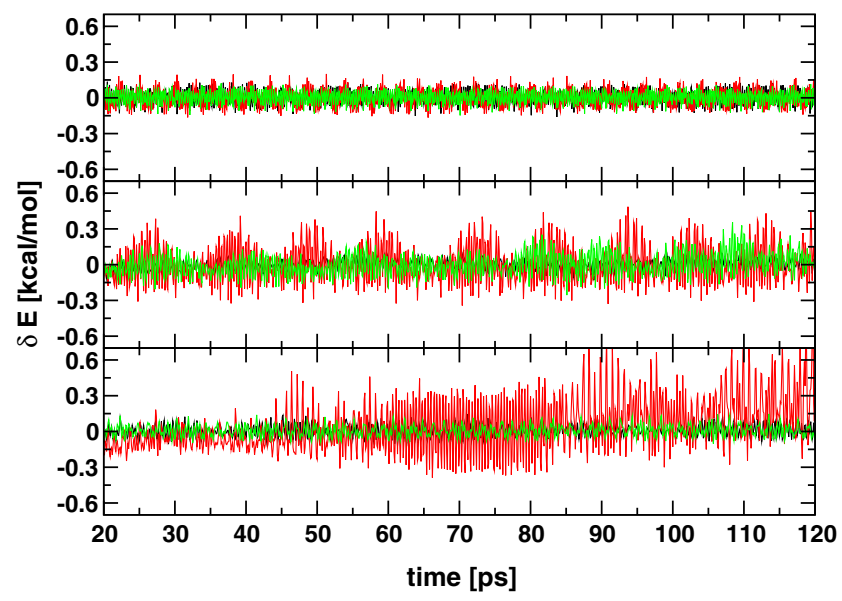

Fig. 7 Energy conservation $(\delta E=E-\bar{E})$ of water/CO cluster during $100 \mathrm{ps}$ at $5 \mathrm{~K}$. Top Only one $\mathrm{CO}$ free to move, centre one $\mathrm{CO}$ and one water free to move, bottom one $\mathrm{CO}$ and two waters free to move. The different colours represent different multipole models on the CO molecules: B (black), C (red), D (green)

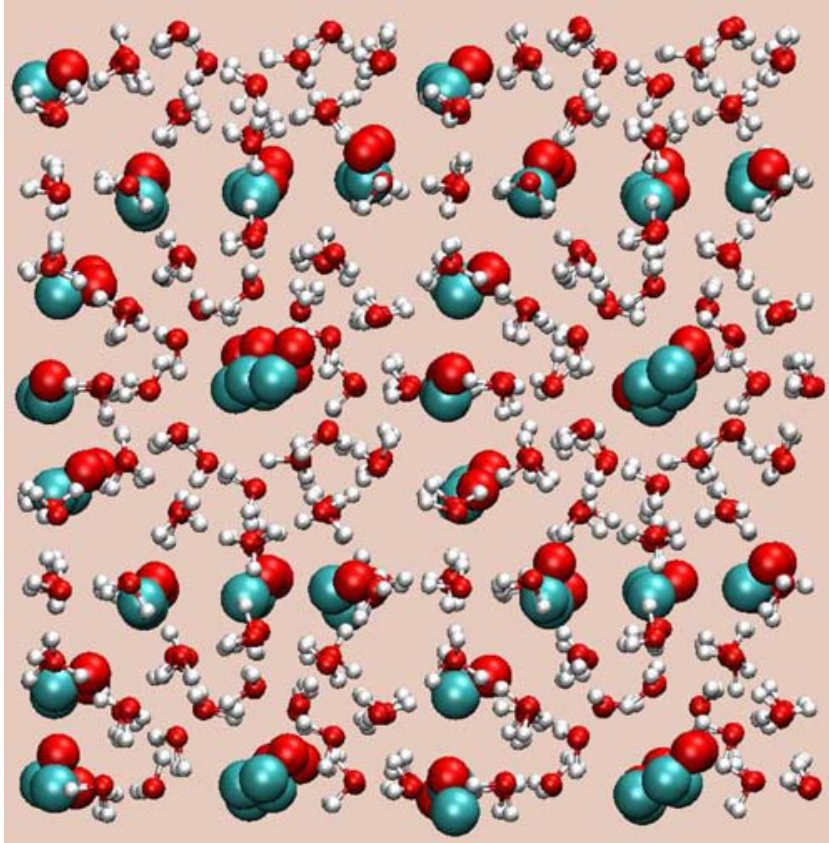

Fig. $8 \mathrm{CO}$ clathrate system to illustrate energy conservation in a $2 \times 2 \times 2$ unit cell of a CO clathrate hydrate

free to move (Fig. 7). For models B and D, these changes leave the energy conservation nearly unaffected. For model $\mathrm{C}$, the changes destabilise the trajectory. These examples show that seemingly small changes in the simulation conditions can affect the stability of the trajectories. The energy change between two conformations of the system and for each atom may be small. Nevertheless, it can become

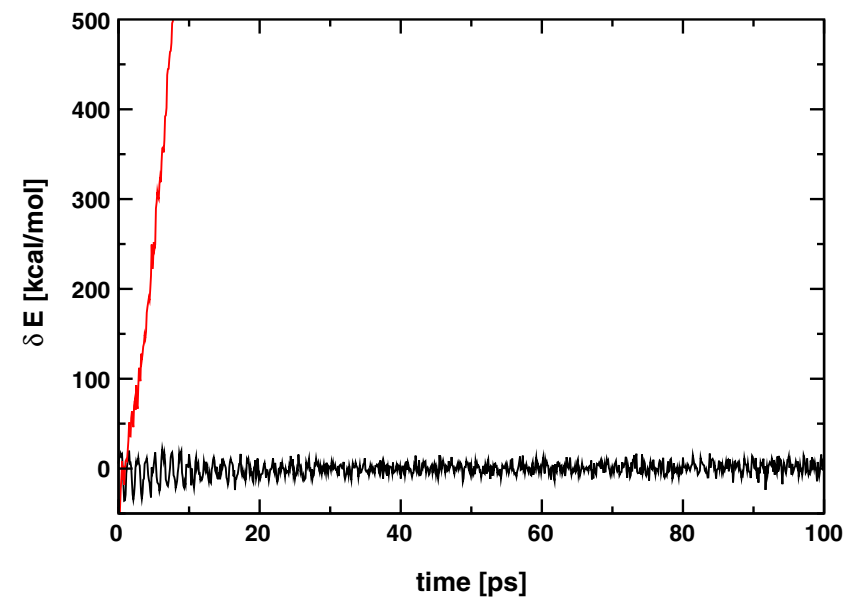

Fig. 9 Energy conservation of $\mathrm{CO}$ clathrate during $100 \mathrm{ps}$ at $100 \mathrm{~K}$. Black CO positions fixed, waters free to move, no energy correction term. Red All molecules free to move, no correction term. The energy values are absolute values of the total energy at each step minus the average total energy during trajectory two [in the case of trajectory two (red), the average would be meaningless, therefore the average of trajectory three (green) is subtracted for comparison] 
very important due to self-amplification. This is explained through the fact that energy changes affect only the rotational energy, which increases due to the rotation of the atomic multipole moments. If the rotational energy increases, the orientational change of the multipole moments also increases. Thus, once the error in energy exceeds a certain threshold, it increases exponentially.

To demonstrate this effect, trajectories are shown for a CO clathrate system (Fig. 8), which consists of water molecules arranged in an ice-like structure around $\mathrm{CO}$ molecules [14]. For the technical evaluation here, water is described by the TIP3P water model and for $\mathrm{CO}$ model $\mathrm{C}$ is used. If the $\mathrm{CO}$ atoms are fixed and the waters move in the field of the atomic multipole expansions, the total energy is conserved because no reorientation of the atomic multipoles takes place. If the $\mathrm{CO}$ molecules are free to move, the energy starts to drift and the self-amplifying effect mentioned above appears (see Fig. 9).

\section{Conclusions}

In conclusion, the present work has shown that atom centred multipole moments are a useful way forward to generalise and improve the description of electrostatic interactions for MD simulations. Technically, the implementation is more involved than for point charges, since the orientation of the multipole moments has to be taken into account. The orientation of the multipole moments can be described through reference axis systems. For rigid molecules, a satisfactory solution that conserves the total energy has been recently presented in the literature [11]. However, for fully flexible molecules, no general solution is as yet available. A generalised implementation for fully flexible molecules requires particular care in order to ensure that total energy is conserved in the simulations. For cases in which the performance of conformationally dependent multipoles with flexible molecules has been carefully examined, clear advantages over point charges in the calculated observables have been found $[15,16]$. Overall, the accuracy of the force field is improved by including higher multipole moments. For larger molecules, the conformational dependence of multipole moments has to be considered and becomes potentially important to maintain the accuracy higher multipole moments can provide.

Acknowledgement The authors gratefully acknowledge financial support from the Swiss National Science Foundation.

\section{References}

1. Bader RFW (1990) Atoms in molecules; a quantum theory. Clarendon, Oxford

2. Besler BH, Merz KM, Kollman PA (1990) J Comp Chem 11:431439

3. Coombes DS, Price SL, Willock DJ, Leslie M (1996) J Phys Chem 100:7352-7360

4. Frisch MJ, Trucks GW, Schlegel HB, Scuseria GE, Robb MA, Cheeseman JR, Montgomery JA Jr, Vreven T, Kudin KN, Burant JC, Millam JM, Iyengar SES, Tomasi J, Barone V, Mennucci B, Cossi M, Scalmani G, Rega N, Petersson GA, Nakatsuji H, Hada M, Ehara M, Toyota K, Fukuda R, Hasegawa J, Ishida M, Nakajima T, Honda Y, Kitao O, Nakai H, Klene M, Li X, Knox JE, Hratchian HP, Cross JB, Adamo C, Jaramillo J, Gomperts R, Stratmann RE, Yazyev O, Austin AJ, Cammi R, Pomelli C, Ochterski JW, Ayala PY, Morokuma K, Voth GA, Salvador P, Dannenberg J, Zakrzewski VG, Dapprich S, Daniels AD, Strain MC, Farkas O, Malick DK, Rabuck AD, Raghavachari K, Foresman JB, Ortiz JV, Cui Q, Baboul AG, Clifford S, Cioslowski J, Stefanov BB, Liu G, Liashenko A, Piskorz P, Komaromi I, Martin RL, Fox DJ, Keith T, Al-Laham MA, Peng CY, Nanayakkara A, Challacombe M, Gill PMW, Johnson B, Chen W, Wong MW, Gonzalez C, Pople JA (2004) Gaussian 03, revision c.01. Gaussian, Inc., Wallingford CT

5. Gray AE, Day GM, Leslie M, Price SL (2004) Mol Phys 102:1067-1083

6. Jorgensen WL, Chandrasekhar JD, Madura JD, Impey RW, Klein ML (1983) J Chem Phys 79:926

7. Joubert L, Popelier PLA (2002) Mol Phys 100:3357-3365

8. Karamertzanis PG, Price SL (2006) J Chem Theo Comp 2:1184-1199

9. Kedzierski P, Sokalski WA (2001) J Comp Chem 22:1082-1097

10. Koch U, Popelier PLA, Stone AJ (1995) Chem Phys Lett 238:253-260

11. Leslie M (2008) Mol Phys 106:1567-1578

12. MacKerell AD Jr, Bashford D, Bellott M, Dunbrack RL Jr, Evanseck JD, Field MJ, Fischer S, Gao J, Guo H, Ha S, JosephMcCarthy D, Kuchnir L, Kuczera K, Lau FTK, Mattos C, Michnick S, Ngo T, Nguyen DT, Prodhom B, Reiher WE III, Roux B, Schlenkrich M, Smith JC, Stote R, Straub JE, Watanabe M, Wiorkiewicz-Kuczera J, Yin D, Karplus M (1998) J Phys Chem B 102:3586-3616

13. Mulliken RS (1955) J Chem Phys 23:1833-1840

14. Plattner N, Bandi T, Doll JD, Freeman DL, Meuwly M (2008) Mol Phys 106:1675-1684

15. Plattner N, Meuwly M (2008a) Chem Phys Chem 9:1271-1277

16. Plattner N, Meuwly M (2008b) Biophys J 94:2505-2515

17. Price SL (1996) J Chem Soc Faraday Trans 92:2997-3008

18. Price SL, Stone AJ (1992) JCSF 88:1755-1763

19. Ren P, Ponder JW (2002) J Comput Chem 23:14971506

20. Singh UC, Kollman PA (1984) J Comput Chem 5:129-145

21. Sokalski WA, Poirier RA (1983) Chem Phys Lett 98:86-92

22. Stone AJ (1981) Chem Phys Lett 83:233-239

23. Stone AJ (1996) The theory of intermolecular forces. Clarendon, Oxford

24. Stone AJ (2005) J Chem Theor Comp 1:1128-1132

25. Stone AJ, Alderton M (1985) Mol Phys 56:1047-1064

26. Weiner SJ, Kollman PA, Case DA, Singh UC, Ghio C, Alagona G, Profeta S Jr, Weiner P (1984) J Am Chem Soc 106:765 